\title{
Effective Secrecy: Reliability, Confusion and Stealth
}

\author{
Jie Hou and Gerhard Kramer \\ Institute for Communications Engineering \\ Technische Universität München, 80290 Munich, Germany \\ Email: \{jie.hou, gerhard.kramer\}@tum.de
}

\begin{abstract}
A security measure called effective security is defined that includes strong secrecy and stealth communication. Effective secrecy ensures that a message cannot be deciphered and that the presence of meaningful communication is hidden. To measure stealth we use resolvability and relate this to binary hypothesis testing. Results are developed for wire-tap channels and broadcast channels with confidential messages.
\end{abstract}

\section{INTRODUCTION}

Wyner [1] derived the secrecy capacity for degraded wiretap channels (see Fig. 11). Csiszár and Körner [2] extended the results to broadcast channels with confidential messages. In both [1] and [2], secrecy was measured by a normalized mutual information between the message $M$ and the eavesdropper's output $Z^{n}$ under a secrecy constraint

$$
\frac{1}{n} I\left(M ; Z^{n}\right) \leq S
$$

which is referred to as weak secrecy. Weak secrecy has the advantage that one can trade off $S$ for rate. The drawback is that even $S \approx 0$ is usually considered too weak because the eavesdropper can decipher $n S$ bits of $M$, which grows with $n$. Therefore, [3] (see also [4]) advocated using strong secrecy where secrecy is measured by the unnormalized mutual information $I\left(M ; Z^{n}\right)$ and requires

$$
I\left(M ; Z^{n}\right) \leq \xi
$$

for any $\xi>0$ and sufficiently large $n$.

In related work, Han and Verdú [5] studied resolvability based on variational distance that addresses the number of bits needed to mimic a marginal distribution of a prescribed joint distribution. Bloch and Laneman [6] used the resolvability approach of [5] and extended the results in [2] to continuous random variables and channels with memory.

The main contribution of this work is to define a new and stronger security measure for wire-tap channels that includes not only reliability and (wiretapper) confusion but also stealth. The measure is satisfied by random codes and by using a recently developed simplified proof [7] of resolvability based on unnormalized informational divergence (see also [8, Lemma 11]). In particular, we measure secrecy by the informational divergence

$$
D\left(P_{M Z^{n}} \| P_{M} Q_{Z}^{n}\right)
$$

where $P_{M Z^{n}}$ is the joint distribution of $M Z^{n}, P_{M}$ is the distribution of $M, P_{Z^{n}}$ is the distribution of $Z^{n}$, and $Q_{Z}^{n}$ is the distribution that the eavesdropper expects to observe when the source is not communicating useful messages. We call this security measure effective secrecy. One can easily check that (see (7) below)

$$
D\left(P_{M Z^{n}} \| P_{M} Q_{Z}^{n}\right)=\underbrace{I\left(M ; Z^{n}\right)}_{\text {Non-Confusion }}+\underbrace{D\left(P_{Z^{n}} \| Q_{Z}^{n}\right)}_{\text {Non-Stealth }}
$$

where we interpret $I\left(M ; Z^{n}\right)$ as a measure of "nonconfusion" and $D\left(P_{Z^{n}} \| Q_{Z}^{n}\right)$ as a measure of "non-stealth". We justify the former interpretation by using error probability in Sec. III and the latter by using binary hypothesis testing in Sec. IV. Thus, by making $D\left(P_{M Z^{n}} \| P_{M} Q_{Z}^{n}\right) \rightarrow 0$ we not only keep the message secret from the eavesdropper but also hide the presence of meaningful communication.

The paper is organized as follows. In Section II] we state the problem. In Section III we state and prove the main result. Section IV relates the result to hypothesis testing. Section $\mathrm{V}$ discusses related works.

\section{PREliminaries}

\section{A. Notation}

Random variables are written with upper case letters and their realizations with the corresponding lower case letters. Superscripts denote finite-length sequences of variables/symbols, e.g., $X^{n}=X_{1}, \ldots, X_{n}$. Subscripts denote the position of a variable/symbol in a sequence. For instance, $X_{i}$ denotes the $i$-th variable in $X^{n}$. We use $X_{i}^{n}$ to denote the sequence $X_{i}, \ldots, X_{n}, 1 \leq i \leq n$. A random variable $X$ has probability distribution $P_{X}$ and the support of $P_{X}$ is denoted as $\operatorname{supp}\left(P_{X}\right)$. We write probabilities with subscripts $P_{X}(x)$ but we drop the subscripts if the arguments of the distribution are lower case versions of the random variables. For example, we write $P(x)=P_{X}(x)$. If the $X_{i}, i=1, \ldots, n$, are independent and identically distributed (i.i.d.) according to $P_{X}$, then we have $P\left(x^{n}\right)=\prod_{i=1}^{n} P_{X}\left(x_{i}\right)$ and we write $P_{X^{n}}=P_{X}^{n}$. We often also use $Q_{X}^{n}$ to refer to sequences of i.i.d. random variables. Calligraphic letters denote sets. The size of a set $\mathcal{S}$ is denoted as $|\mathcal{S}|$ and the complement is denoted as $\mathcal{S}^{\mathrm{c}}$. For $X$ with alphabet $\mathcal{X}$, we denote $P_{X}(\mathcal{S})=\sum_{x \in \mathcal{S}} P_{X}(x)$ for any $\mathcal{S} \subseteq \mathcal{X}$. We use $\mathcal{T}_{\epsilon}^{n}\left(P_{X}\right)$ to denote the set of lettertypical sequences of length $n$ with respect to the probability distribution $P_{X}$ and the non-negative number $\epsilon$ [9, Ch. 3], [10], i.e., we have

$\mathcal{T}_{\epsilon}^{n}\left(P_{X}\right)=\left\{x^{n}:\left|\frac{N\left(a \mid x^{n}\right)}{n}-P_{X}(a)\right| \leq \epsilon P_{X}(a), \forall a \in \mathcal{X}\right\}$ where $N\left(a \mid x^{n}\right)$ is the number of occurrences of $a$ in $x^{n}$. 


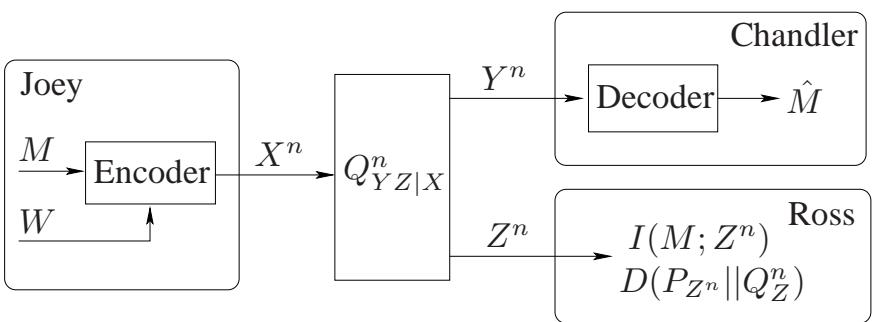

Fig. 1. A wire-tap channel.

\section{B. Wire-Tap Channel}

Consider the wire-tap channel depicted in Fig. 11 Joey has a message $M$ which is destined for Chandler but should be kept secret from Ross. The message $M$ is uniformly distributed over $\{1, \ldots, L\}, L=2^{n R}$, and an encoder $f(\cdot)$ maps $M$ to the sequence

$$
X^{n}=f(M, W)
$$

with help of a randomizer variable $W$ that is independent of $M$ and uniformly distributed over $\left\{1, \ldots, L_{1}\right\}, L_{1}=2^{n R_{1}}$. The purpose of $W$ is to confuse Ross so that he learns little about $M . X^{n}$ is transmitted through a memoryless channel $Q_{Y Z \mid X}^{n}$. Chandler observes the channel output $Y^{n}$ while Ross observes $Z^{n}$. The pair $M Z^{n}$ has the joint distribution $P_{M Z^{n}}$. Chandler estimates $\hat{M}$ from $Y^{n}$ and the average error probability is

$$
P_{e}^{(n)}=\operatorname{Pr}[\hat{M} \neq M] .
$$

Ross tries to learn $M$ from $Z^{n}$ and secrecy is measured by

$$
\begin{aligned}
D & \left(P_{M Z^{n}} \| P_{M} Q_{Z}^{n}\right) \\
& =\sum_{\substack{\left(m, z^{n}\right) \\
\in \operatorname{supp}\left(P_{\left.M Z^{n}\right)}\right.}} P\left(m, z^{n}\right) \log \left(\frac{P\left(m, z^{n}\right)}{P(m) \cdot Q_{Z}^{n}\left(z^{n}\right)} \cdot \frac{P\left(z^{n}\right)}{P\left(z^{n}\right)}\right) \\
& =\sum_{\substack{\left(m, z^{n}\right) \\
\in \operatorname{supp}\left(P_{\left.M Z^{n}\right)}\right.}} P\left(m, z^{n}\right)\left(\log \frac{P\left(z^{n} \mid m\right)}{P\left(z^{n}\right)}+\log \frac{P\left(z^{n}\right)}{Q_{Z}^{n}\left(z^{n}\right)}\right) \\
& =\underbrace{I\left(M ; Z^{n}\right)}_{\text {Non-Confusion }}+\underbrace{D\left(P_{Z^{n}} \| Q_{Z}^{n}\right)}_{\text {Non-Stealth }}
\end{aligned}
$$

where $P_{Z^{n}}$ is the distribution Ross observes at his channel output and $Q_{Z}^{n}$ is the distribution Ross expects to observe if Joey is not sending useful information. For example, if Joey transmits $X^{n}$ with probability $Q_{X}^{n}\left(X^{n}\right)$ through the channel, then we have

$$
Q_{Z}^{n}\left(z^{n}\right)=\sum_{x^{n} \in \operatorname{supp}\left(Q_{X}^{n}\right)} Q_{X}^{n}\left(x^{n}\right) Q_{Z \mid X}^{n}\left(z^{n} \mid x^{n}\right) .
$$

When Joey sends useful messages, then $P_{Z^{n}}$ and $Q_{Z}^{n}$ are different. But a small $D\left(P_{M Z^{n}} \| P_{M} Q_{Z}^{n}\right)$ implies that both $I\left(M ; Z^{n}\right)$ and $D\left(P_{Z^{n}} \| Q_{Z}^{n}\right)$ are small which in turn implies that Ross learns little about $M$ and cannot recognize whether Joey is communicating anything meaningful. A rate $R$ is achievable if for any $\xi_{1}, \xi_{2}>0$ there is a sufficiently large $n$ and an encoder and a decoder such that

$$
\begin{aligned}
P_{e}^{(n)} & \leq \xi_{1} \\
D\left(P_{M Z^{n}} \| P_{M} Q_{Z}^{n}\right) & \leq \xi_{2} .
\end{aligned}
$$

The effective secrecy capacity $C_{S}$ is the supremum of the set of achievable $R$. We wish to determine $C_{S}$.

\section{MAin RESUlt AND PRoOF}

We prove the following result.

Theorem 1: The effective secrecy capacity of the wire-tap channel is the same as the weak and strong secrecy capacity, namely

$$
C_{S}=\max _{Q_{V X}}[I(V ; Y)-I(V ; Z)]
$$

where the maximization is over all joint distributions $Q_{V X}$ satisfying the Markov chain

$$
V-X-Y Z \text {. }
$$

One may restrict the cardinality of $V$ to $|\mathcal{V}| \leq|\mathcal{X}|$.

\section{A. Achievability}

We use random coding and the proof technique of [7].

Random Code: Fix a distribution $Q_{X}$ and generate $L \cdot L_{1}$ codewords $x^{n}(m, w), m=1, \ldots, L, w=1, \ldots, L_{1}$ using $\prod_{i=1}^{n} Q_{X}\left(x_{i}(m, w)\right)$. This defines the codebook

$$
\mathcal{C}=\left\{x^{n}(m, w), m=1, \ldots, L, w=1, \ldots, L_{1}\right\}
$$

and we denote the random codebook by

$$
\widetilde{\mathcal{C}}=\left\{X^{n}(m, w)\right\}_{(m, w)=(1,1)}^{\left(L, L_{1}\right)} .
$$

Encoding: To send a message $m$, Joey chooses $w$ uniformly from $\left\{1, \ldots, L_{1}\right\}$ and transmits $x^{n}(m, w)$. Hence, for a fixed codebook $\mathcal{C}$ every $x^{n}(m, w)$ occurs with probability

$$
P_{X^{n}}\left(x^{n}(m, w)\right)=\frac{1}{L \cdot L_{1}}
$$

rather than $Q_{X}^{n}\left(x^{n}(m, w)\right)$. Further, for every pair $\left(m, z^{n}\right)$ we have (see (8))

$$
\begin{aligned}
P\left(z^{n} \mid m\right) & =\sum_{w=1}^{L_{1}} \frac{1}{L_{1}} \cdot Q_{Z \mid X}^{n}\left(z^{n} \mid x^{n}(m, w)\right) \\
P\left(z^{n}\right) & =\sum_{m=1}^{L} \sum_{w=1}^{L_{1}} \frac{1}{L \cdot L_{1}} \cdot Q_{Z \mid X}^{n}\left(z^{n} \mid x^{n}(m, w)\right) .
\end{aligned}
$$

Chandler: Chandler puts out $(\hat{m}, \hat{w})$ if there is a unique pair $(\hat{m}, \hat{w})$ satisfying the typicality check

$$
\left(x^{n}(\hat{m}, \hat{w}), y^{n}\right) \in \mathcal{T}_{\epsilon}^{n}\left(Q_{X Y}\right) .
$$

Otherwise he puts out $(\hat{m}, \hat{w})=(1,1)$.

Analysis: Define the events

$$
\begin{aligned}
& E_{1}:\{(\hat{M}, \hat{W}) \neq(M, W)\} \\
& E_{2}: D\left(P_{M Z^{n}} \| P_{M} Q_{Z}^{n}\right)>\xi_{2} .
\end{aligned}
$$

Let $E=E_{1} \cup E_{2}$ so that we have

$$
\operatorname{Pr}[E] \leq \operatorname{Pr}\left[E_{1}\right]+\operatorname{Pr}\left[E_{2}\right]
$$

where we have used the union bound. $\operatorname{Pr}\left[E_{1}\right]$ can be made small with large $n$ as long as

$$
R+R_{1}<I(X ; Y)-\delta_{\epsilon}(n)
$$


where $\delta_{\epsilon}(n) \rightarrow 0$ as $n \rightarrow \infty$ (see [10]) which implies that $P_{e}^{(n)}$ is small.

$\operatorname{Pr}\left[E_{2}\right]$ can be made small with large $n$ as long as [7. Theorem 1]

$$
R_{1}>I(X ; Z)+\delta_{\epsilon}^{\prime}(n)
$$

where $\delta_{\epsilon}^{\prime}(n) \rightarrow 0$ as $n \rightarrow \infty$. This is because the average divergence over $M, W, \widetilde{\mathcal{C}}$ and $Z^{n}$ satisfies (see [7, Equ. (9)])

$$
\begin{aligned}
& \mathrm{E}\left[D\left(P_{M Z^{n}} \| P_{M} Q_{Z}^{n}\right)\right] \\
& \stackrel{(a)}{=} \mathrm{E}\left[D\left(P_{M}|| P_{M}\right)+D\left(P_{Z^{n} \mid M}|| Q_{Z}^{n} \mid P_{M}\right)\right] \\
& \stackrel{(b)}{=} \mathrm{E}\left[\log \frac{\sum_{j=1}^{L_{1}} Q_{Z \mid X}^{n}\left(Z^{n} \mid X^{n}(M, j)\right)}{L_{1} \cdot Q_{Z}^{n}\left(Z^{n}\right)}\right] \\
& =\sum_{m=1}^{L} \sum_{w=1}^{L_{1}} \frac{1}{L \cdot L_{1}} \\
& \mathrm{E}\left[\log \frac{\sum_{j=1}^{L_{1}} Q_{Z \mid X}^{n}\left(Z^{n} \mid X^{n}(m, j)\right) \mid}{L_{1} \cdot Q_{Z}^{n}\left(Z^{n}\right)} \mid M=m, W=w\right] \\
& \stackrel{(c)}{\leq} \sum_{m=1}^{L} \sum_{w=1}^{L_{1}} \frac{1}{L \cdot L_{1}} \\
& \mathrm{E}\left[\log \left(\frac{Q_{Z \mid X}^{n}\left(Z^{n} \mid X^{n}(m, w)\right)}{L_{1} \cdot Q_{Z}^{n}\left(Z^{n}\right)}+1\right) \mid M=m, W=w\right] \\
& \stackrel{(d)}{=} \mathrm{E}\left[\log \left(\frac{Q_{Z \mid X}^{n}\left(Z^{n} \mid X^{n}\right)}{L_{1} \cdot Q_{Z}^{n}\left(Z^{n}\right)}+1\right)\right]
\end{aligned}
$$

where

(a) follows from the chain rule for informational divergence;

(b) follows from (16) and by taking the expectation over $M, W, X^{n}(1,1), \ldots, X^{n}\left(L, L_{1}\right), Z^{n}$

(c) follows by the concavity of the logarithm and Jensen's inequality applied to the expectation over the $X^{n}(m, j), j \neq$ $w$ for a fixed $m$;

(d) follows by choosing $X^{n} Z^{n} \sim Q_{X Z}^{n}$.

Next we can show that the right hand side (RHS) of (23) is small if 22 is valid by splitting the expectation in 23 into sums of typical and atypical pairs (see [7] Equ. (11)-(16)]). But if the RHS of (23) approaches 0 , then using (7) we have

$$
\mathrm{E}\left[I\left(M ; Z^{n}\right)+D\left(P_{Z^{n}} \| Q_{Z}^{n}\right)\right] \rightarrow 0
$$

Combining 201, 21 and we can make $\operatorname{Pr}[E] \rightarrow 0$ as $n \rightarrow \infty$ as long as

$$
\begin{array}{r}
R+R_{1}<I(X ; Y) \\
R_{1}>I(X ; Z) .
\end{array}
$$

We hence have the achievability of any $R$ satisfying

$$
0 \leq R<\max _{Q_{X}}[I(X ; Y)-I(X ; Z)]
$$

Of course, if the RHS of 27 is non-positive, then we require $R=0$. Now we prefix a channel $Q_{X \mid V}^{n}$ to the original channel
$Q_{Y Z \mid X}^{n}$ and obtain a new channel $Q_{Y Z \mid V}^{n}$ where

$$
\begin{aligned}
& Q_{Y Z \mid V}^{n}\left(y^{n}, z^{n} \mid v^{n}\right) \\
& =\sum_{x^{n} \in \operatorname{supp}\left(Q_{X \mid V}^{n}\left(\cdot \mid v^{n}\right)\right)} Q_{X \mid V}^{n}\left(x^{n} \mid v^{n}\right) Q_{Y Z \mid X}^{n}\left(y^{n}, z^{n} \mid x^{n}\right) .
\end{aligned}
$$

Using a similar analysis as above, we have the achievability of any $R$ satisfying

$$
0 \leq R<\max _{Q_{V X}}[I(V ; Y)-I(V ; Z)]
$$

where the maximization is over all $Q_{V X}$ satisfying (12). Again, if the RHS of 29) is non-positive, then we require $R=0$. As usual, the purpose of adding the auxiliary variable $V$ is to potentially increase $R$. Note that $V=X$ recovers (27). Hence, the RHS of 27 is always smaller than or equal to the RHS of 29.

Remark 1: The average divergence $\mathrm{E}\left[D\left(P_{M Z^{n}} \| P_{M} Q_{Z}^{n}\right)\right]$ can be viewed as the sum of $I\left(M \widetilde{\mathcal{C}} ; Z^{n}\right)$ and $D\left(P_{Z^{n}} \| Q_{Z}^{n}\right)$ [11, Sec. III] (see also [7, Sec. III-B]). To see this, consider

$$
\begin{aligned}
\mathrm{E} & {\left[D\left(P_{M Z^{n}} \| P_{M} Q_{Z}^{n}\right)\right] } \\
& \stackrel{(a)}{=} \mathrm{E}\left[\log \frac{\sum_{j=1}^{L_{1}} Q_{Z \mid X}^{n}\left(Z^{n} \mid X^{n}(M, j)\right)}{L_{1} \cdot Q_{Z}^{n}\left(Z^{n}\right)}\right] \\
= & \sum_{m=1}^{L} \frac{1}{L} \sum_{x^{n}(1,1)} \ldots \sum_{x^{n}\left(L, L_{1}\right)} \prod_{k=(1,1)} Q_{X}^{n}\left(x^{n}(k)\right) \\
& \sum_{z^{n}} \sum_{w=1}^{L_{1}} \frac{1}{L_{1}} Q_{Z \mid X}^{n}\left(z^{n} \mid x^{n}(m, w)\right) \\
& \log \left[\frac{\sum_{j=1}^{L_{1}} \frac{1}{L_{1}} Q_{Z \mid X}^{n}\left(z^{n} \mid x^{n}(m, j)\right)}{Q_{Z}^{n}\left(z^{n}\right)}\right] \\
= & \sum_{m=1}^{L} P(m) \sum_{\mathcal{C}} P(\mathcal{C} \mid m) \sum_{z^{n}} P\left(z^{n} \mid m, \mathcal{C}\right) \log \frac{P\left(z^{n} \mid m, \mathcal{C}\right)}{Q_{Z}^{n}\left(z^{n}\right)} \\
= & \sum_{\left(m, \mathcal{C}, z^{n}\right)} P\left(m, \mathcal{C}, z^{n}\right)\left(\log \frac{P\left(z^{n} \mid m, \mathcal{C}\right)}{P\left(z^{n}\right)}+\log \frac{P\left(z^{n}\right)}{Q_{Z}^{n}\left(z^{n}\right)}\right) \\
= & I\left(M \mathcal{C} ; Z^{n}\right)+D\left(P_{Z^{n}} \| Q_{Z}^{n}\right)
\end{aligned}
$$

where (a) follows by 23) (b). Therefore, as $\mathrm{E}\left[D\left(P_{M Z^{n}} \| P_{M} Q_{Z}^{n}\right)\right] \rightarrow 0$ we have $I\left(M \widetilde{\mathcal{C}} ; Z^{n}\right) \rightarrow 0$ which means that $M \widetilde{\mathcal{C}}$ and $Z^{n}$ are (almost) independent. This makes sense, since for effective secrecy the adversary learns little about $M$ nor about the presence of meaningful transmission.

\section{B. Converse}

The converse follows as in [2, Theorem 1]. We provide an alternative proof using the telescoping identity [12, Sec. G]. Suppose that for some $\xi_{1}, \xi_{2}>0$ there exists a sufficiently large $n$, an encoder and a decoder such that (9) and (10) are 
satisfied. We have

$$
\begin{aligned}
& \log _{2} L=n R \\
& =H(M) \\
& =I\left(M ; Y^{n}\right)+H\left(M \mid Y^{n}\right) \\
& \stackrel{(a)}{\leq} I\left(M ; Y^{n}\right)+\left(1+\xi_{1} \cdot n R\right) \\
& \stackrel{(b)}{\leq} I\left(M ; Y^{n}\right)-I\left(M ; Z^{n}\right)+\xi_{2}+\left(1+\xi_{1} \cdot n R\right)
\end{aligned}
$$

where $(a)$ follows from Fano's inequality and $(b)$ follows from (7) and (10). Using the telescoping identity [12, Equ. (9) and (11)] we have

$$
\begin{aligned}
& \frac{1}{n}\left[I\left(M ; Y^{n}\right)-I\left(M ; Z^{n}\right)\right] \\
& =\sum_{i=1}^{n}\left[I\left(M ; Z_{i+1}^{n} Y^{i}\right)-I\left(M ; Z_{i}^{n} Y^{i-1}\right)\right] \\
& =\frac{1}{n} \sum_{i=1}^{n}\left[I\left(M ; Y_{i} \mid Y^{i-1} Z_{i+1}^{n}\right)-I\left(M ; Z_{i} ; \mid Y^{i-1} Z_{i+1}^{n}\right)\right] \\
& \stackrel{(a)}{=} I\left(M ; Y_{T} \mid Y^{T-1} Z_{T+1}^{n} T\right)-I\left(M ; Z_{T} \mid Y^{T-1} Z_{T+1}^{n} T\right) \\
& \stackrel{(b)}{=} I(V ; Y \mid U)-I(V ; Z \mid U) \\
& \leq \max _{Q_{U V X}}[I(V ; Y \mid U)-I(V ; Z \mid U)] \\
& \leq \max _{u} \max _{Q_{V X \mid U=u}}[I(V ; Y \mid U=u)-I(V ; Z \mid U=u)] \\
& \stackrel{(c)}{=} \max _{Q_{V X}}[I(V ; Y)-I(V ; Z)]
\end{aligned}
$$

where

(a) follows by letting $T$ be independent of all other random variables and uniformly distributed over $\{1, \ldots, n\}$;

(b) follows by defining

$$
\begin{aligned}
& U=Y^{T-1} Z_{T+1}^{n} T, V=M U, \\
& X=X_{T}, Y=Y_{T}, Z=Z_{T} ;
\end{aligned}
$$

(c) follows because if the maximum in (32) is achieved for $U=u^{*}$ and $Q_{V X \mid U=u^{*}}$, then the same can be achieved in (33) by choosing a $Q_{V X}=Q_{V X \mid U=u^{*}}$.

Combining (31) and (33) we have

$$
R \leq \frac{\max _{Q_{V X}}[I(V ; Y)-I(V ; Z)]}{1-\xi_{1}}+\frac{\xi_{2}+1}{\left(1-\xi_{1}\right) n} .
$$

Letting $n \rightarrow \infty, \xi_{1} \rightarrow 0$, and $\xi_{2} \rightarrow 0$, we have

$$
R \leq \max _{Q_{V X}}[I(V ; Y)-I(V ; Z)]
$$

where the maximization is over all $Q_{V X}$ satisfying the Markov chain (12). The cardinality bound in Theorem 1 was derived in [13, Theorem 22.1]. This completes the converse.

\section{Broadcast Channels with Confidential Messages}

Broadcast channels with confidential messages (BCC) [2] are wire-tap channels with common messages. For the BCC (Fig. 2), Joey has a common message $M_{0}$ destined for both Chandler and Ross which is independent of $M$ and uniformly

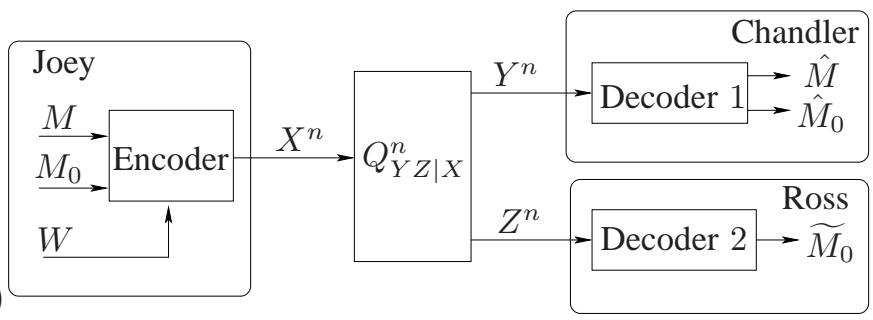

Fig. 2. A broadcast channel with a confidential message.

distributed over $\left\{1, \ldots, L_{0}\right\}, L_{0}=2^{n R_{0}}$. An encoder maps $M_{0}$ and $M$ to

$$
X^{n}=f\left(M_{0}, M, W\right)
$$

which is sent through the channel $Q_{Y Z \mid X}^{n}$. Chandler estimates $\left(\hat{M}_{0}, \hat{M}\right)$ from $Y^{n}$ while Ross estimates $\widetilde{M}_{0}$ from $Z^{n}$. The average error probability is

$$
P_{e}^{*(n)}=\operatorname{Pr}\left[\left\{\left(\hat{M}_{0}, \hat{M}\right) \neq\left(M_{0}, M\right)\right\} \cup\left\{\widetilde{M}_{0} \neq M_{0}\right\}\right]
$$

and non-secrecy is measured by $D\left(P_{M Z^{n}} \| P_{M} Q_{Z}^{n}\right)$. A rate pair $\left(R_{0}, R\right)$ is achievable if, for any $\xi_{1}, \xi_{2}>0$, there is a sufficiently large $n$, an encoder and two decoders such that

$$
\begin{aligned}
P_{e}^{*(n)} & \leq \xi_{1} \\
D\left(P_{M Z^{n}} \| P_{M} Q_{Z}^{n}\right) & \leq \xi_{2} .
\end{aligned}
$$

The effective secrecy capacity region $C_{\mathrm{BCC}}$ is the closure of the set of achievable $\left(R_{0}, R\right)$. We have the following theorem.

Theorem 2: $C_{\mathrm{BCC}}$ is the same as the weak and strong secrecy capacity region

$$
C_{\mathrm{BCC}}=\bigcup\left\{\begin{array}{l}
\left(R_{0}, R\right): \\
0 \leq R_{0} \leq \min \{I(U ; Y), I(U ; Z)\} \\
0 \leq R \leq I(V ; Y \mid U)-I(V ; Z \mid U)
\end{array}\right\}
$$

where the union is over all distributions $Q_{U V X}$ satisfying the Markov chain

$$
U-V-X-Y Z \text {. }
$$

One may restrict the alphabet sizes to

$$
|\mathcal{U}| \leq|\mathcal{X}|+3 ; \quad|\mathcal{V}| \leq|\mathcal{X}|^{2}+4|\mathcal{X}|+3 .
$$

Proof: The proof is omitted due to the similarity to the proof of Theorem 1

\section{Choice of Security Measures}

Effective secrecy includes both strong secrecy and stealth communication. One may argue that using only $I\left(M ; Z^{n}\right)$ or $D\left(P_{Z^{n}} \| Q_{Z}^{n}\right)$ would suffice to measure secrecy. However, we consider two examples where secrecy is achieved but not stealth, and where stealth is achieved but not secrecy.

Example 1: $I\left(M ; Z^{n}\right) \rightarrow 0, D\left(P_{Z^{n}} \| Q_{Z}^{n}\right)=D>0$. Suppose that Joey inadvertently uses $\widetilde{Q}_{X}$ rather than $Q_{X}$ for codebook generation, where (22) is still satisfied. The new $\widetilde{Q}_{X}$ 


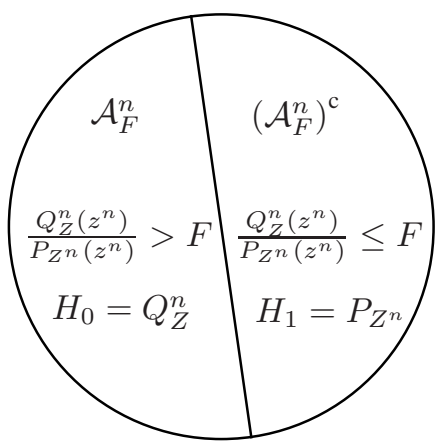

Fig. 3. Example of the decision regions $\mathcal{A}_{F}^{n}$ and $\left(\mathcal{A}_{F}^{n}\right)^{\mathrm{c}}$.

could result in a different expected $\widetilde{Q}_{Z}^{n} \neq Q_{Z}^{n}$. Hence, as $n$ grows large we have

$$
D\left(P_{M Z^{n}} \| P_{M} Q_{Z}^{n}\right)=I\left(M ; Z^{n}\right)+D\left(\widetilde{Q}_{Z}^{n} \| Q_{Z}^{n}\right)
$$

where $I\left(M ; Z^{n}\right) \rightarrow 0$ but we have

$$
D\left(\widetilde{Q}_{Z}^{n} \| Q_{Z}^{n}\right)=D \text {, for some } D>0 .
$$

Ross thus recognizes that Joey is transmitting useful information even though he cannot decode.

Example 2: $I\left(M ; Z^{n}\right)=I>0, D\left(P_{Z^{n}} \| Q_{Z}^{n}\right) \rightarrow 0$.

Note that $\mathrm{E}\left[D\left(P_{Z^{n}} \| Q_{Z}^{n}\right)\right] \rightarrow 0$ as $n \rightarrow \infty$ as long as (see [7. Theorem 1])

$$
R+R_{1}>I(X ; Z) .
$$

If Joey is not careful and chooses $R_{1}$ such that 22 is violated and (46) is satisfied, then $D\left(P_{Z^{n}} \| Q_{Z}^{n}\right)$ can be made small but we have

$$
I\left(M ; Z^{n}\right)=I \text { for some } I>0 .
$$

Thus, although the communication makes $D\left(P_{Z^{n}} \| Q_{Z}^{n}\right)$ small, Ross can learn

$$
I\left(M ; Z^{n}\right) \approx n\left[I(X ; Z)-R_{1}\right]
$$

bits about $M$ if he is willing to pay a price and always tries to decode (see Sec. IV]).

\section{Hypothesis Testing}

The reader may wonder how $D\left(P_{Z^{n}} \| Q_{Z}^{n}\right)$ relates to stealth. We consider a hypothesis testing framework and show that as long as (46) is satisfied, the best Ross can do to detect Joey's action is to guess.

For every channel output $z^{n}$, Ross considers two hypotheses

$$
\begin{aligned}
& H_{0}=Q_{Z}^{n} \\
& H_{1}=P_{Z^{n}} .
\end{aligned}
$$

If $H_{0}$ is accepted, then Ross decides that Joey's transmission is not meaningful, whereas if $H_{1}$ is accepted, then Ross decides that Joey is sending useful messages. We define two kinds of error probabilities

$$
\begin{aligned}
& \alpha=\operatorname{Pr}\left\{H_{1} \text { is accepted } \mid H_{0} \text { is true }\right\} \\
& \beta=\operatorname{Pr}\left\{H_{0} \text { is accepted } \mid H_{1} \text { is true }\right\} .
\end{aligned}
$$

The value $\alpha$ is referred to as the level of significance [14] and corresponds to the probability of raising a false alarm, while $\beta$ corresponds the probability of mis-detection. In practice, raising a false alarm can be expensive. Therefore, Ross would like to minimize $\beta$ for a given tolerance level of $\alpha$. To this end, Ross performs for every $z^{n}$ a ratio test

$$
\frac{Q_{Z}^{n}\left(z^{n}\right)}{P_{Z^{n}}\left(z^{n}\right)}=r
$$

and makes a decision depending on a threshold $F, F \geq 0$, namely

$$
\left\{\begin{array}{ll}
H_{0} \text { is accepted } & \text { if } r>F \\
H_{1} \text { is accepted } & \text { if } r \leq F
\end{array} .\right.
$$

Define the set of $z^{n}$ for which $H_{0}$ is accepted as

$$
\mathcal{A}_{F}^{n}=\left\{z^{n}: \frac{Q_{Z}^{n}\left(z^{n}\right)}{P_{Z^{n}}\left(z^{n}\right)}>F\right\}
$$

and $\left(\mathcal{A}_{F}^{n}\right)^{\mathrm{c}}$ is the set of $z^{n}$ for which $H_{1}$ is accepted (see Fig. 3). Ross chooses the threshold $F$ and we have

$$
\begin{aligned}
\alpha & =Q_{Z}^{n}\left(\left(\mathcal{A}_{F}^{n}\right)^{\mathrm{c}}\right)=1-Q_{Z}^{n}\left(\mathcal{A}_{F}^{n}\right) \\
\beta & =P_{Z^{n}}\left(\mathcal{A}_{F}^{n}\right) .
\end{aligned}
$$

The ratio test in (53) is the Neyman-Pearson test which is optimal [14, Theorem 3.2.1] in the sense that it minimizes $\beta$ for a given $\alpha$. We have the following lemma.

Lemma 1: If $D\left(P_{Z^{n}} \| Q_{Z}^{n}\right) \leq \xi_{2}, \xi_{2}>0$, then with the Neyman-Pearson test we have

$$
1-g\left(\xi_{2}\right) \leq \alpha+\beta \leq 1+g\left(\xi_{2}\right)
$$

where

$$
g\left(\xi_{2}\right)=\sqrt{\xi_{2} \cdot 2 \ln 2}
$$

which goes to 0 as $\xi_{2} \rightarrow 0$.

Proof: Since $D\left(P_{Z^{n}} \| Q_{Z}^{n}\right) \leq \xi_{2}$, we have (see 60)

$$
\left\|P_{Z^{n}}-Q_{Z}^{n}\right\|_{\mathrm{TV}} \leq \sqrt{\xi_{2} \cdot 2 \ln 2}=g\left(\xi_{2}\right)
$$

where

$$
\left\|P_{X}-Q_{X}\right\|_{\mathrm{TV}}=\sum_{x \in \mathcal{X}}|P(x)-Q(x)|
$$

is the variational distance between $P_{X}$ and $Q_{X}$ and where the inequality follows by Pinsker's inequality [15, Theorem 11.6.1]. We further have

$$
\begin{aligned}
& \left\|P_{Z^{n}}-Q_{Z}^{n}\right\|_{\mathrm{TV}} \\
& =\sum_{z^{n} \in \mathcal{A}_{F}^{n}}\left|P_{Z^{n}}\left(z^{n}\right)-Q_{Z}^{n}\left(z^{n}\right)\right| \\
& +\sum_{z^{n} \in\left(\mathcal{A}_{F}^{n}\right)^{\mathrm{c}}}\left|P_{Z^{n}}\left(z^{n}\right)-Q_{Z}^{n}\left(z^{n}\right)\right| \\
& \geq \sum_{z^{n} \in \mathcal{A}_{F}^{n}}\left|P_{Z^{n}}\left(z^{n}\right)-Q_{Z}^{n}\left(z^{n}\right)\right| \\
& \stackrel{(a)}{\geq}\left|\sum_{z^{n} \in \mathcal{A}_{F}^{n}}\left[P_{Z^{n}}\left(z^{n}\right)-Q_{Z}^{n}\left(z^{n}\right)\right]\right| \\
& =\left|P_{Z^{n}}\left(\mathcal{A}_{F}^{n}\right)-Q_{Z}^{n}\left(\mathcal{A}_{F}^{n}\right)\right| \\
& =|\beta-(1-\alpha)|
\end{aligned}
$$




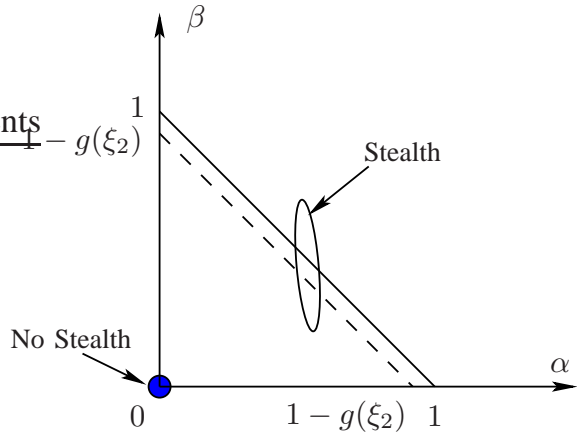

Fig. 4. Optimal tradeoff between $\alpha$ and $\beta$.

where (a) follows by the triangle inequality. Combining (59) and 61 , we have the bounds 57.

Fig. 4 illustrates the optimal tradeoff between $\alpha$ and $\beta$ for stealth communication, i.e., when (46) is satisfied. As $n \rightarrow \infty$ and $\xi_{2} \rightarrow 0$, we have

$$
\begin{aligned}
D\left(P_{Z^{n}} \| Q_{Z}^{n}\right) & \rightarrow 0 \\
(\alpha+\beta) & \rightarrow 1 .
\end{aligned}
$$

If Ross allows no false alarm $(\alpha=0)$, then he always ends up with mis-detection $(\beta=1)$. If Ross tolerates no mis-detection $(\beta=0)$, he pays a high price $(\alpha=1)$. Further, for any given $\alpha$, the optimal mis-detection probability is

$$
\beta_{\text {opt }}=1-\alpha .
$$

But Ross does not need to see $Z^{n}$ or perform an optimal test to achieve $\beta_{\text {opt }}$. He may randomly choose some $\mathcal{A}^{\prime}$ such that

$$
Q_{Z}^{n}\left(\left(\mathcal{A}^{\prime}\right)^{\mathrm{c}}\right)=\alpha
$$

and achieves $\beta_{\mathrm{opt}}^{\prime}=1-\alpha$. The best strategy is thus to guess. On the other hand, if

$$
\lim _{n \rightarrow \infty} D\left(P_{Z^{n}} \| Q_{Z}^{n}\right)>0
$$

then Ross detects Joey's action and we can have

$$
\alpha+\beta=0 \text {. }
$$

We thus operate in one of two regimes in Fig. 4, either near $(\alpha, \beta)=(0,0)$ or near the line $\alpha+\beta=1$.

\section{Discussion}

Our resolvability proof differs from that in [6] in that we rely on unnormalized informational divergence [7] instead of variational distance [5]. Our proof is simpler and the result is stronger than that in [6] when restricting attention to product distributions and memoryless channels because a small $D\left(P_{M Z^{n}} \| P_{M} Q_{Z}^{n}\right)$ implies small $I\left(M ; Z^{n}\right)$ and $D\left(P_{Z^{n}} \| Q_{Z}^{n}\right)$ while a small $\left\|P_{X^{n}}-Q_{X}^{n}\right\|_{\mathrm{TV}}$ implies only a small $I\left(M ; Z^{n}\right)$ [4. Lemma 1].

Hayashi studied strong secrecy for wire-tap channels using resolvability based on unnormalized divergence and he derived bounds for nonasymptotic cases [11, Theorem 3]. We remark that Theorem 1 can be derived by extending [11, Lemma 2] to asymptotic cases. However, Hayashi did not consider stealth but focused on strong secrecy, although he too noticed a formal connection to (7) [11, p. 1568].

\section{ACKNOWLEDGMENT}

J. Hou and G. Kramer were supported by an Alexander von Humboldt Professorship endowed by the German Federal Ministry of Education and Research. G. Kramer was also supported by NSF Grant CCF-09-05235. J. Hou thanks Rafael Schaefer for useful discussions.

\section{REFERENCES}

[1] A. Wyner, "The wire-tap channel," Bell Syst. Tech. Journal, vol. 54, no. 8, pp. $1355-1387$, Oct. 1975.

[2] I. Csiszár and J. Körner, "Broadcast channels with confidential messages," IEEE Trans. Inf. Theory, vol. 24, no. 3, pp. 339-348, May 1978.

[3] U. Maurer and S. Wolf, "Information-theoretic key agreement: From weak to strong secrecy for free," in Advances in Cryptology - Eurocrypt 2000. Lecture Notes in Computer Science, Springer-Verlag, 2000, pp. 351-368.

[4] I. Csiszár, "Almost independence and secrecy capacity," Prob. Inf. Trans., vol. 32, no. 1, pp. 40-47, Jan.-March 1996.

[5] T. Han and S. Verdú, "Approximation theory of output statistics," IEEE Trans. Inf. Theory, vol. 39, no. 3, pp. 752-772, May 1993.

[6] M. Bloch and N. Laneman, "Strong secrecy from channel resolvability," IEEE Trans. Inf. Theory, vol. 59, no. 12, pp. 8077-8098, Dec. 2013.

[7] J. Hou and G. Kramer, "Informational divergence approximations to product distributions," in 13th Canadian Workshop on Inf. Theory (CWIT), Toronto, Canada, June 2013, pp. 76-81.

[8] A. Winter, "Secret, public and quantum correlation cost of triples of random variables," in IEEE Int. Symp. Inf. Theory, Adelaide, Australia, Sept. 2005, pp. 2270-2274.

[9] J. L. Massey, Applied Digital Information Theory, ETH Zurich, Zurich, Switzerland, 1980-1998.

[10] A. Orlitsky and J. Roche, "Coding for computing," IEEE Trans. Inf. Theory, vol. 47, no. 3, pp. 903-917, March 2001.

[11] M. Hayashi, "General nonasymptotic and asymptotic formulas in channel resolvability and identification capacity and their application to the wiretap channel," IEEE Trans. Inf. Theory, vol. 52, no. 4, pp. 15621575, April 2006

[12] G. Kramer, "Teaching IT: An identity for the Gelfand-Pinsker converse," IEEE Inf. Theory Society Newsletter, vol. 61, no. 4, pp. 4-6, Dec. 2011.

[13] A. El Gamal and Y.-H. Kim, Network Information Theory. Cambridge University Press, 2011

[14] E. L. Lehmann and J. P. Romano, Testing Statistical Hypotheses, 3rd ed. New York: Springer, 2005.

[15] T. Cover and J. Thomas, Elements of Information Theory, 2nd ed. New York: Wiley, 2006. 